\section{Echocardiographic Detection of Pericardiocentesis-Induced Subepicardial and Intramyocardial Hematoma}

Jon N. Meliones, MD, A. Rebecca Snider, MD, Robert H. Beekman, MD, A. Resai Bengur, MD, and Michele A. Bogaards, MD

- ericardiocentesis has been widely used to relieve significant pericardial effusion and, in most cases, can be performed safely and without complications. We describe a rare complication of pericardiocentesis not previously reported in a pe- diatric patient. The crucial role of $2-$ dimensional echocardiography in the detection of this rare complication is illustrated.

$R B$ was hospitalized at 3 months of age for failure to thrive, hydrocephalus and dysmorphism. On ad- mission, the infant's weight was 3.08 $\mathrm{kg}(<$ fifth percentile), his hemoglobin was $8.7 \mathrm{~g} / \mathrm{dl}$, and he had severe diarrhea and vomiting. On the tenth hospital day, $R B$ developed septice. mia and Candida parapsilosis was grown from blood cultures. Therapy was begun with amphotericin $B$ and

From the Department of Pediatrics, F1609, C.S. Mott Children's Hospital, University of Michigan Medical Center, Ann Arbor, Michigan 48109-0204. Manuscript received June 19,1989 ; revised manuscript received and accepted July 13, 1989.

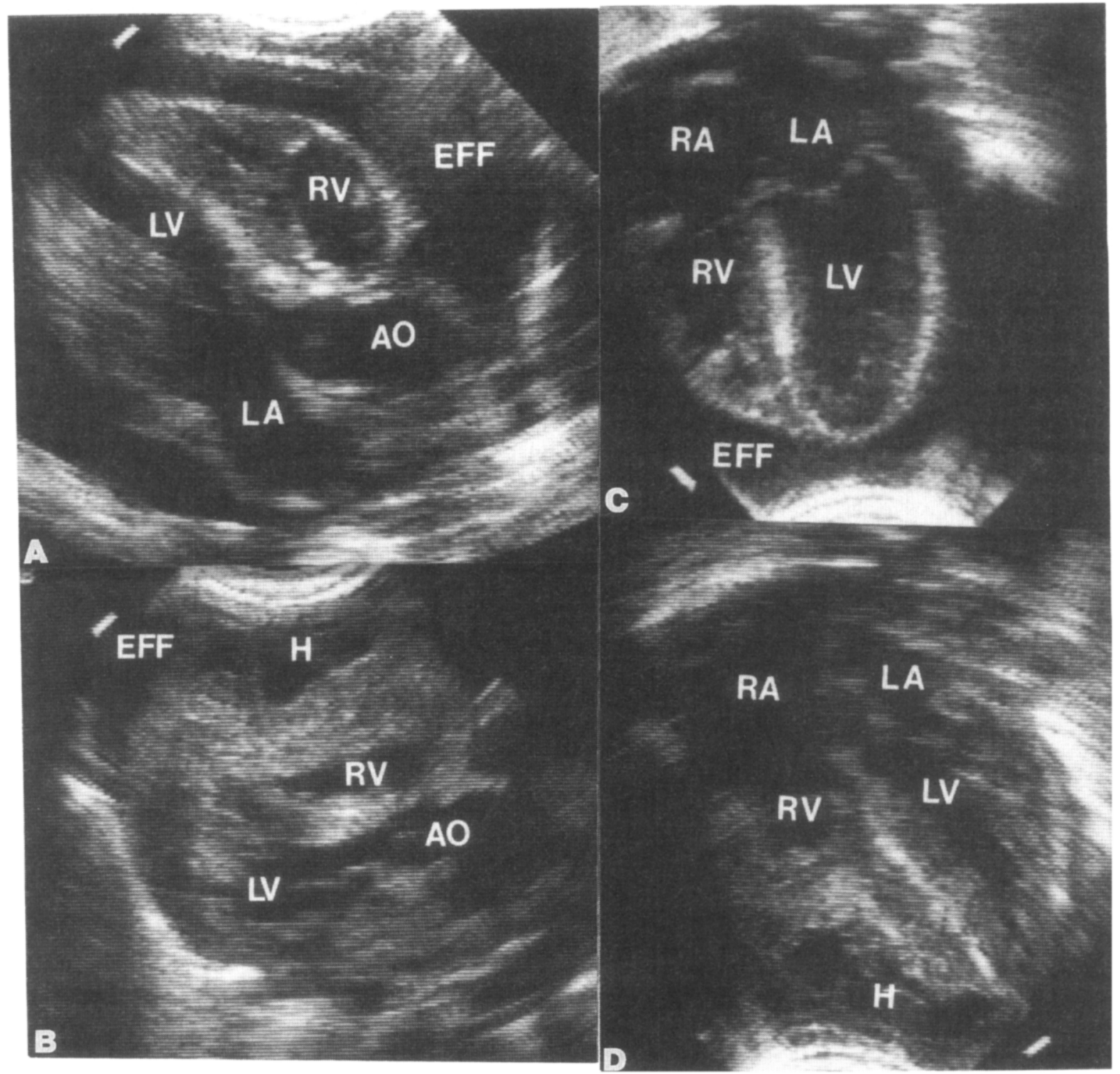

FGURE 1. Two-dmensional echocardographic images from the parastemal long-axls vew before (A) and afier (B) pericardio-

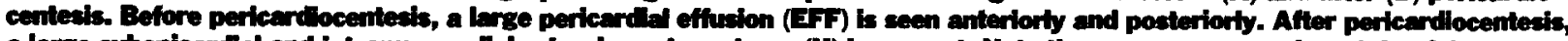
a large subopicardal and intramyocard echo-dense hematoma (U) is present. Note the severe compreacion of the right ventricular cavity by the hematoma. Two-dmenciond echocardocraphic images from the apical 4-chamber view before (C) and after (D) pericandiocentesis. Before the pericardiocentects, a large pericerdilal effusion (EFF) is noted. Afler pericardiocentesis, a large echo-dense hematoma (H) is seen in the diaphragmatic surface of the right ventuicle (RV) extending to the cardiac apex. There is near total obiteration of the RV cavity by the hematoma. AO = acrta; LA = left atrinm; LV = left ventricle; $\mathbf{R A}=$ right afrim. 
5-fluorocytosine. Despite therapy, respiratory distress worsened and the infant was intubated on the twenty-fourth hospital day. Serial chest roentgenograms demonstrated an increasing cardiothoracic silhouette, and a 2-dimensional echocardiogram performed on the twentyeighth hospital day showed a moderate pericardial effusion. On the thirty-third hospital day, RB developed acidosis, increased central venous pressure, increased heart size and decreased perfusion. An emergent 2-dimensional echocardiogram demonstrated a large pericardial effusion with tamponade (Figure 1).

Pericardiocentesis was performed from a subxiphoid approach with a 16-gauge needle. Sixty-two ml of straw-colored fluid was withdrawn without difficulty. Shortly after the procedure, the infant developed a heart rate of 200 beats/min, central venous pressure of $20 \mathrm{~mm}$ $\mathrm{Hg}$ and blood pressure of $60 / 45 \mathrm{~mm}$ Hg. A chest roentgenogram showed a right pneumothorax, which was relieved by placement of a chest tube. An electrocardiogram showed evidence of myocardial injury with massive elevation of the $S T$-T wave segments in the anterior chest leads. An emergent 2-dimensional echocardiogram was performed; it showed an echo-dense fluid mass within the myocardium of the right ventricle (Figure 1). The fluid mass involved the anterior and diaphragmatic surfaces of the right ventricle and was associated with severe right ventricular compression. The infant was taken immediately to the operating room where a large subepicardial and intramyocardial hematoma was found in the anterior and diaphragmatic walls of the right ventricle. The hematoma was evacuated and a pericardial tube was placed. Despite relief of the tamponade and inotropic support, the infant died approximately 2 hours after surgery. At autopsy, a subepicardial and intramyocardial hematoma was confirmed.

Pericardiocentesis can be difficult in infants and this case represents a rare complication not previously reported in a pediatric patient. A review of previous reports disclosed 4 adult patients with this unusual complication..$^{1-4}$ The use of echocardiography for the diagnosis of pericardial effusion and for guidance of the nee- dle during pericardiocentesis has been well described. ${ }^{5}$ Our case illustrates that echocardiography can also provide important information concerning the results and complications of pericardiocentesis. In any patient with deterioration after pericardiocentesis, 2-dimensional echocardiography provides a technique for the rapid, noninvasive diagnosis of complications. This application of $2-$ dimensional echocardiography is especially important in the critically ill, unstable infant.

1. Schuster AH, Nanda NC. Pericardiocentesis induced intraepicardial thrombus: detection by twodimensional echocardiography. Am Heart $J$ 1982; 104:308-311.

2. Preis LK, Taylor GJ, Martin RP. Traumatic pericardiocentesis: two dimensional echocardiographic visualization of an unfortunate event. Arch Intern Med 1982:142:2327-2329.

3. Lin CS, Jan YI, Chen HY, Hou SH, Kuo CC. Two-dimensional echocardiographic detection of pericardiocentesis induced intrapericardial thrombus. Chest 1984;86:787-789.

4. Calabrese P, Iliceto $S$, Rizzon P. Pericardiocentesis induced intraepicardial thrombus: visualization of thrombus formation and spontaneous internal lysis by two-dimensional echocardiography. J Clin Ultrasound 1985;13:49-51.

5. Callahan JA, Seward JB, Nishimura RA, Miller FA Jr, Reeder GS, Shub C, Callahan MJ, Schattenberg TT, Tajik $\Lambda$ J. Two-dimensional cehocardiographic guided pericardiocentesis: experience in 117 consecutive patients. Am J Cardiol 1985;55:476 479. 